\title{
Problemática de los recubrimientos superficiales realizados con láser e influencia de los parámetros de proceso
}

\author{
M. Cadenas ${ }^{(*)}$, J.M. Cuetos ${ }^{(*)}$, E. Fernández-Rico ${ }^{(*)}$, J.M. Sierra ${ }^{(*)}$
}

\begin{abstract}
Resumen Los recubrimientos depositados sobre la superficie de un substrato, mediante la inyección en un haz láser de un material en forma de polvo, pueden tener importantes ventajas frente a recubrimientos depositados con otras técnicas, tales como una disminución apreciable de la porosidad, un amplio intervalo de espesores de capa, una mayor dureza, tratamiento en áreas localizadas, etc. En este artículo se presenta la problemática con la que el grupo de trabajo de los autores se ha encontrado al utilizar esta técnica: velocidad de las partículas inadecuada, inestabilidad del caudal de polvo, efecto del ángulo de inyección y alteraciones sobre el flujo de polvo provocadas por el gas de protección de la óptica de focalización del láser. También se presentan las soluciones adoptadas y los cambios y mejoras que éstos han supuesto para el resultado final de las capas. Asimismo, se analiza la influencia de los parámetros del proceso en las características geométricas del recubrimiento (altura del cordón y de la zona afectada térmicamente, penetración, ancho del cordón, etc.) y en la dilución. El objetivo final es contribuir en la medida de lo posible al conocimiento de esta técnica.
\end{abstract}

Palabras clave: Recubrimientos superficiales. Parámetros láser. Carburo de volframio. Carburo de cromo.

\section{Problems of laser cladding technique and influence of the laser processing parameters}

\begin{abstract}
The coatings deposited on the surface of a substrate of a material in powder form, by injection in a laser beam, they could have important advantages opposite coatings deposited with other technical. These advantages are a very important decrease of the porosity, an interval of thickness of wide covering, a higher coating hardness, treatment in located areas, etc. In this paper our investigation group presents the problems found utilising this technique: inadequate speed of the particles, unstableness of the powder flow rate, effect of the angle of injection and alterations on the powder flow caused by the protective gas of the laser focusing head. We also present the adopted solutions and the improvements that these solutions have supposed for the properties of the coatings. The influence of the laser processing parameters in the dilution and in the characteristics of the coating is also studied (height of the track, penetration, etc.). Our final objective is to contribute to the knowledge of this technique.
\end{abstract}

Keywords: Laser cladding. Laser parameters. Tungsten carbide. Chromium carbide.

\section{INTRODUCCIÓN}

El recubrimiento superficial con láser consiste en inyectar partículas calibradas de polvo en medio de la radiación fotónica de un láser, de manera que estas partículas interaccionen con los fotones una

(*) Área de Ingeniería Mecánica. E.T.S. I.I. Univ. de Oviedo. Campus de Viesques, s/n. 33203-Gijón (Asturias, España) Tlf.: (98) 51824 19. Fax (98) 51820 60. E-mail: cadenas@sci.cpd.uniovi.es. distancia controlada, produciéndose su fusión y depósito sobre la superficie del substrato. Con ello, se obtiene una capa de la aleación inyectada, con una cierta dilución que varía en función de los parámetros utilizados (1).

Su posible interés industrial, frente a otros métodos alternativos, tales como la proyección por plasma o la soldadura por arco, reside en el hecho de ser uno de los tratamientos superficiales que causan menor daño térmico y distorsión al substrato, se consiguen muy buenas adherencias capa-substrato y 
espesores de capa variables en un amplio rango. Además, se pueden producir nuevas microestructuras, improbables con otras técnicas, debido a la rápida solidificación y a la alta concentración de elementos en la superficie, y una reducción de la porosidad del depósito y de la dilución.

Esta técnica resulta adecuada para el tratamiento de pequeñas áreas, tales como asientos de válvulas, bordes de álabes de turbina, superficies de desgaste de herramientas, etc. (2). Para grandes áreas, puede ser rentable si se consigue que el proceso se autocorrija de los efectos de calentamiento en el substrato y si se domina el problema de esfuerzos internos inducidos en el material (3).

\section{PROCEDIMIENTO EXPERIMENTAL}

El equipamiento utilizado consiste en un equipo láser de $\mathrm{CO}_{2}$ Rofin Sinar de $1.700 \mathrm{~W}$ de potencia nominal, con una distribución transversal de energía en forma de "donut" $\left(\mathrm{TEM}_{01 *}\right)$.

La inyección del polvo se ha realizado fuera del cabezal focalizador del láser. Para ello, se utilizó finalmente un inyector a $60^{\circ}$, de $2,5 \mathrm{~mm}$ de diámetro interior, al que le llega el polvo arrastrado por gas argón a 3 bar, a través de una manguera flexible, desde un alimentador de polvo Metco 4MP. Se trata de un equipo especialmente diseñado para procesos de proyección por plasma, pero para utilizarlo con láser resultó poco adecuado, puesto que el caudal de polvo necesario (entre 5 y $25 \mathrm{~g} / \mathrm{min}$ ) siempre estaba en el rango de los caudales mínimos que podía suministrar, lo que provocaba cierta inestabilidad en el mismo, cuando el proceso depende en gran medida de un caudal de polvo uniforme.

Se trabajó con los siguientes materiales en forma de polvo, típicos de la industria de obtención de recargues mediante otras técnicas:

- Una mezcla al $50 \%$ del cermet $88 \%$ WCCo y una aleación metálica de $\mathrm{NiCrFe}$, de 40 $\mu \mathrm{m}$ de tamaño medio de grano (Metco 34F).

- Un cermet $83 \%$ WC-Co y $39 \mu \mathrm{m}$ de tamaño medio (Metco 73F-NS-1).

- Un cermet $75 \%$ CrC-NiCr y $22 \mu \mathrm{m}$ de tamaño medio (Metco 81VF-NS).

\section{PROBLEMÁTICA Y SOLUCIONES}

El objetivo es conseguir capas con características tales como dureza, resistencia a la corrosión, al desgaste, etc., con mínima dilución y un espesor entre 0,3 y $1 \mathrm{~mm}$ (que depende de los parámetros utilizados), con buena adherencia con el substrato y sin grietas ni poros (4). Para ello, se han ido superando diversos problemas:
- Presencia de grietas en los recubrimientos realizados con los materiales descritos anteriormente, sin que se hayan podido evitar. Precalentar el substrato, añadir elementos metálicos a los polvos, y bajas velocidades y/o bajas densidades de potencia pueden reducir el problema del agrietamiento.

- Problemas de porosidad, que aunque es escasa, en algunos casos, los poros son de gran tamaño. Su origen puede estar en cavidades entre dos cordones solapados, mala limpieza del substrato o a causa del desprendimiento de gases disueltos, como ocurre en la soldadura convencional. La realización del recubrimiento superficial con láser en cámara de vacío puede solucionar este problema. En cualquier caso, hemos constatado que el ajuste de los parámetros del proceso permite disminuir la mayor parte de la misma.

- Problemas relacionados con el sistema de inyección de polvos

- No es indiferente trabajar en una dirección o en otra, debido a la falta de simetría respecto del haz láser. Los mejores resultados se obtienen inyectando el polvo por delante del haz con respecto al sentido del desplazamiento (1).

- El ángulo de inyección tiene importancia para el alineamiento entre el flujo de polvo y el haz láser, y para el tiempo que el polvo va a estar sometido a radiación láser (Fig. 1). Para ángulos pequeños (< $45^{\circ}$ con la horizontal), el polvo proyectado estará menos tiempo dentro del haz y tendrá más facilidad de rebotar y perderse (1). Los inyectores con ángulos mayores de $45^{\circ}$, favorecen la obtención de cordones de mayor espesor, más homogéneos y de menor porosidad.

- Para minimizar las pérdidas por el rebote de las partículas de polvo sobre la superficie, es imprescindible reducir su
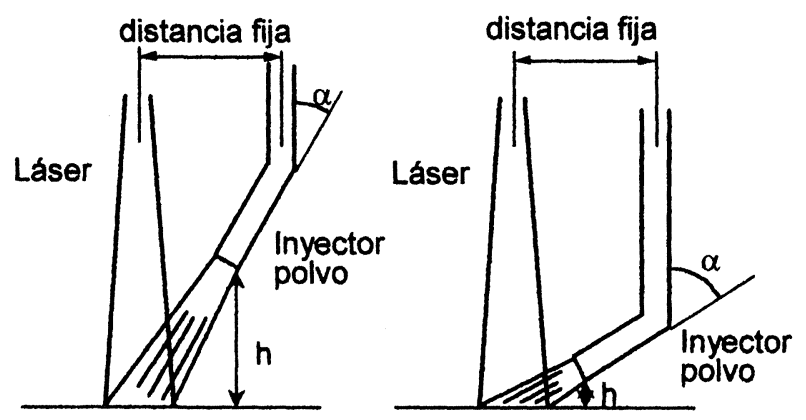

FIG. 1.- Influencia del ángulo del inyector.

FIG. 1.- Influence of the angle of powder nozzle. 
velocidad de salida del inyector. Por ello, a lo largo del inyector, se practicó una serie de orificios de purga de $1 \mathrm{~mm}$ de diámetro (hasta un total de 25). La presión de entrada del gas de arrastre al alimentador se tomó constante e igual a 3 bares.

- Perturbación de los gases de protección del cabezal focalizador del láser sobre el chorro de inyección del polvo, lo que provoca el soplado y la dispersión del polvo inyectado. También influye en la correcta alineación de la corriente de polvo y el haz láser, ya que la posición del inyector se debe ajustar de tal forma que cuando confluyen los dos flujos de gas, el polvo incida en el lugar deseado.

Para resolver este problema, se utilizó un segundo inyector orientado horizontalmente, situado justo a la salida de la boquilla del cabezal del láser (Fig. 2), que proporciona una corriente de aire comprimido a una presión de 0,5 bar, suficiente para barrer totalmente el flujo de gas de la boquilla. Con ello, se consigue que el polvo inyectado no se disperse y que se obtenga un mayor rendimiento. También contribuye a mejorar la porosidad del recubrimiento y a disminuir en gran medida la fusión del substrato, mejorando la dilución y aumentando su dureza (5).

En la figura 3 se pueden ver dos cordones de WC-Co sobre un acero F-1140 realizados con los mismos parámetros. La única diferencia entre ambos es que uno se ha realizado sin la corriente de

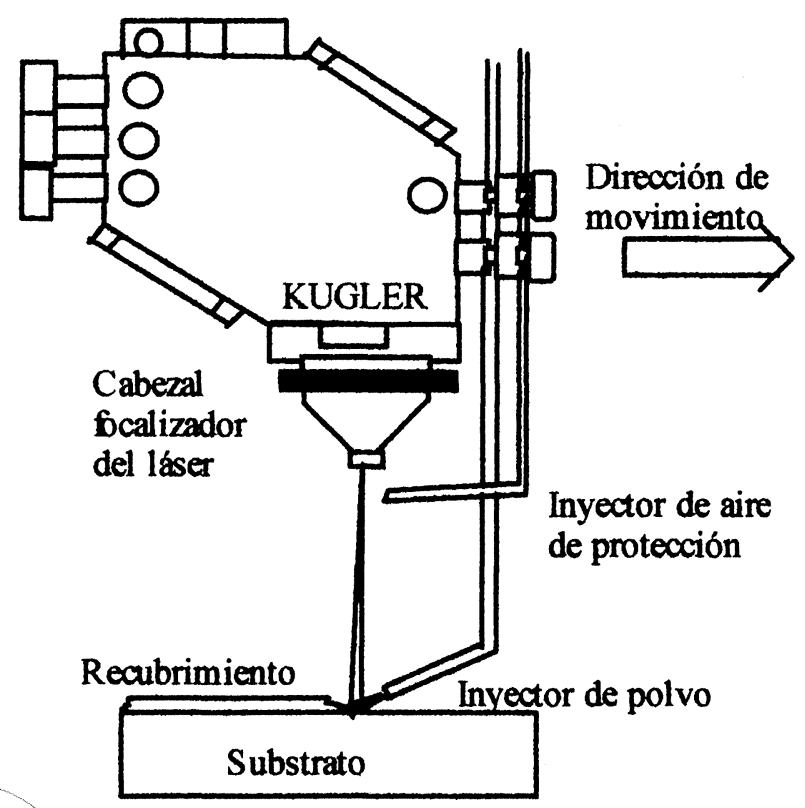

FIG. 2.- Disposición para realizar recubrimientos superficiales con láser.

FIG. 2.- Assembling used to laser cladding tests.

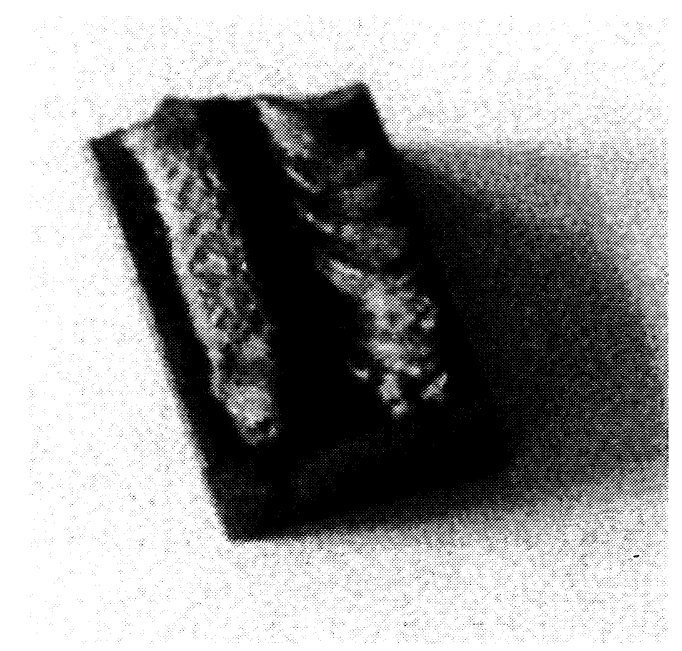

FIG. 3.- Cordones de WC-Co realizados con y sin aire de protección.

FIG. 3.- WC-Co laser cladded tracks with and without air of protection.

aire horizontal (el de peor aspecto) y el otro con ella.

\section{VARIABLES DEL PROCESO Y SU INFLUENCIA}

En la tabla I se muestran las variables de operación que ha sido necesario manejar. El gran número de variables a tener en cuenta, da una idea de la dificultad que entraña encontrar los parámetros más adecuados para cada material, ya que cada uno influye de una forma determinada y, a su vez, se ve influido por otras variables. Así, por ejemplo, el parámetro que más influye en el espesor de la capa es la velocidad de procesado; sin embargo, el caudal de polvo y el solapamiento entre los distintos cordones también influyen en éste de una forma importante. Del mismo modo, resulta ser el caudal de polvo el que más influye en la dilución, pero ésta también depende de las otras variables.

Se pueden unir el caudal y la velocidad de proceso en una única variable denominada "cantidad de polvo aportado por unidad de longitud", definida como:

$$
m_{p}(\mathrm{~g} / \mathrm{cm})=\frac{\text { caudal }(\mathrm{g} / \mathrm{min})}{\operatorname{velocidad}(\mathrm{cm} / \mathrm{min})}
$$

Esta variable recoge los efectos que provocan las variaciones de caudal y velocidad, independizándonos de ellos. Los gráficos de la figura 4 representan la geometría de un cordón de $\mathrm{WC}-\mathrm{Co} / \mathrm{NiCr}-$ Fe en función de dicha variable para una energía 
TABLA I.- Variables del proceso de recubrimiento con láser.

TABLE I.- Variables of laser cladding process.

\begin{tabular}{|l|l|l|}
\hline \multicolumn{1}{|c|}{ LÁSER } & \multicolumn{1}{|c|}{ INYECCIÓN DE POLVOS } & \multicolumn{1}{c|}{ MATERIAL } \\
\hline Potencia (P) & Tipo de inyector & Material de recubrimiento \\
Diámetro del haz (D) & Ángulo del inyector & tipo de probeta \\
Densidad de potencia $\left(4 \mathrm{P} / \pi \mathrm{D}^{2}\right)$ & Distancia inyector-pieza & Estado superficial \\
Velocidad de procesado $(\mathrm{V})$ & Alineamiento del polvo con el haz & \\
Gas de protección & Cauda de polvo $(\mathrm{Q})$ & \\
Presión gas protección & Gas arrastre & \\
Distancia de desfocalización & Presión gas arrastre & \\
Tiempo de interacción (D/V) & Densidad de polvo (Q/DV) & \\
Energía específica (P/DV) & Energía por gramo de polvo (P/Q) & \\
Diámetro boquilla & & \\
Solapamiento entre cordones & & \\
\hline
\end{tabular}

específica aportada constante. A mayor $m_{p}$, mayor altura del cordón y menor dilución.

Por otro lado, la cantidad de energía liberada por unidad de masa de polvo adherida al substrato, determina el grado de dilución a obtener (6). En el gráfico de la figura 5 , se puede observar cómo aumenta la dilución con el aumento de energía por masa de polvo aportado (adherido más perdido) y cómo hay una energía por masa de polvo mínima para conseguir dilución cero para un cordón de WC-Co/NiCrFe. Valores inferiores a este mínimo hacen que no haya adherencia del polvo con el substrato.

La dilución, y la microestructura también se ven afectadas por el solapamiento de cordones. Al realizar el solapamiento, una parte del material depositado previamente es nuevamente fundido. Esto da

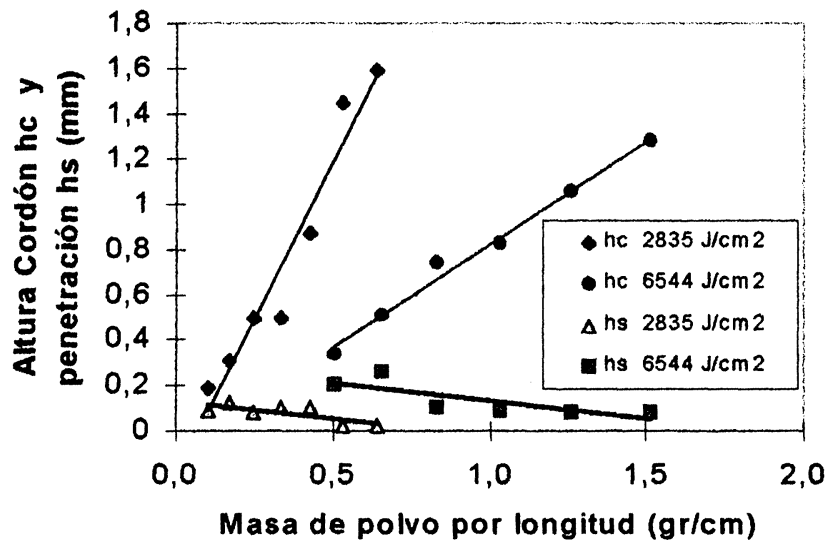

lugar a que en la zona de solapamiento haya una estructura diferente a la del resto de la capa (Fig. 6). En la zona en que hubo una única fusión, los carburos son grandes, de forma prismática; sin embargo, en la zona de solapamiento, los carburos son más pequeños y de perfil redondeado, resultado de una mayor disolución de los mismos. Además, en esta zona y en la de la intercara, el contenido de hierro, procedente de la fusión del substrato, aumenta considerablemente. Este fenómeno asegura la adherencia entre capa y substrato (7), pero cuanto mayor es la dilución (o el contenido de hierro), menor es la dureza de la capa (Fig. 7).

La figura 8 ilustra el fenómeno de la dilución: la figura $8 A$ muestra una capa de $\mathrm{CrC}-\mathrm{NiCr}$ y una fusión elevada del substrato, por lo que el contenido de hierro de aquélla es muy elevado. El ataque

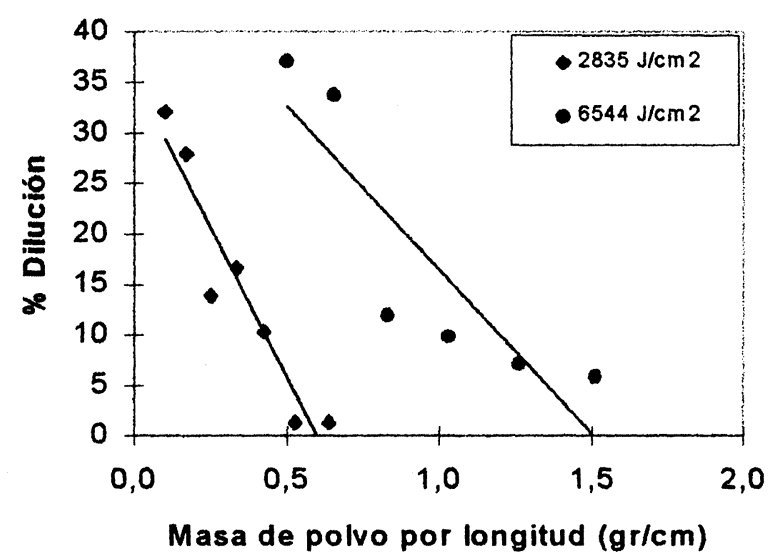

FIG. 4.- Parámetros geométricos de un cordón de WC-Co/NICrFe en función de la masa de polvo aportado por unidad de-longitud.

FIG. 4.- Relationship of geometric parameters (height (hc) and penetration (hs)) of a WC-Co/NiCrFe laser cladded track with the linear mass feed rate $(\mathrm{g} / \mathrm{cm})$ under different specific energy $\left(\mathrm{J} / \mathrm{cm}^{2}\right)$. 


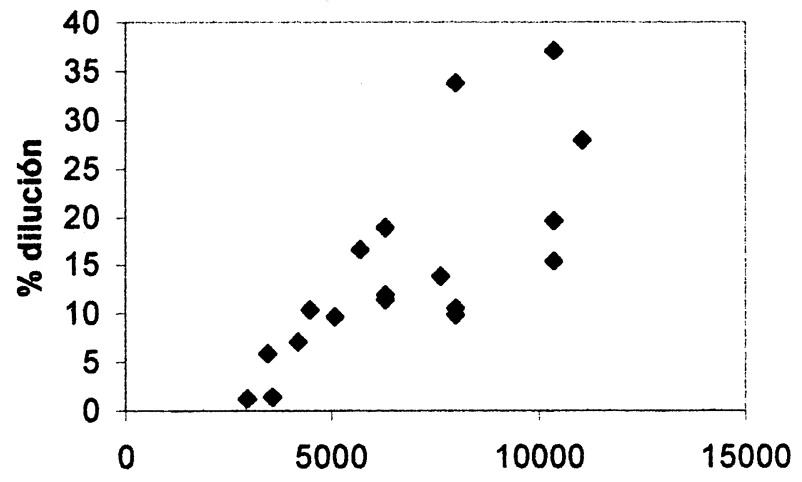

Energía por masa de polvo (Jullos/gramo)

FIG. 5.- Dilución en función de la energía por masa de polvo para un cordón de $83 \%$ WC-Co recargada por laser $\mathrm{WC}-\mathrm{Co} / \mathrm{NiCrFe}$.

FIG. 5.- Relationship of the dilution of a WC$\mathrm{Co} / \mathrm{NiCrFe}$ laser cladded track with the laser energy per mass of powder $(\mathrm{J} / \mathrm{g})$.

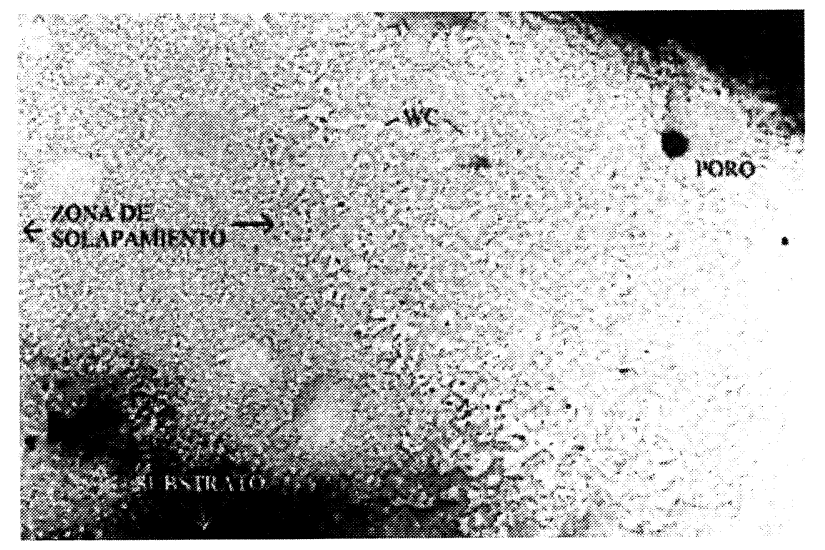

FIG. 6.- Microestructura de las capas de $83 \%$ WC-Co Recargada por laser.

FIG. 6.- Microstructure of $83 \%$ WC-Co laser cladded coating.

químico revela el contenido de hierro mediante una tonalidad clara. Sin embargo, en $8 B$ se ve una capa con poca dilución, donde únicamente en las zonas de solapamiento y en la intercara hay presencia de hierro.

Finalmente, y después de algunos ensayos previos, se acotaron los valores de las variables: caudales de polvo entre 5 y $25 \mathrm{~g} / \mathrm{min}$., densidades de potencia entre 6.000 a $18.000 \mathrm{~W} / \mathrm{cm}^{2}$ y velocidades de procesado desde 200 hasta $800 \mathrm{~mm} / \mathrm{min}$.

\section{CONCLUSIONES}

La técnica de recubrimiento superficial con láser mediante la inyección de partículas fuera del cabe-

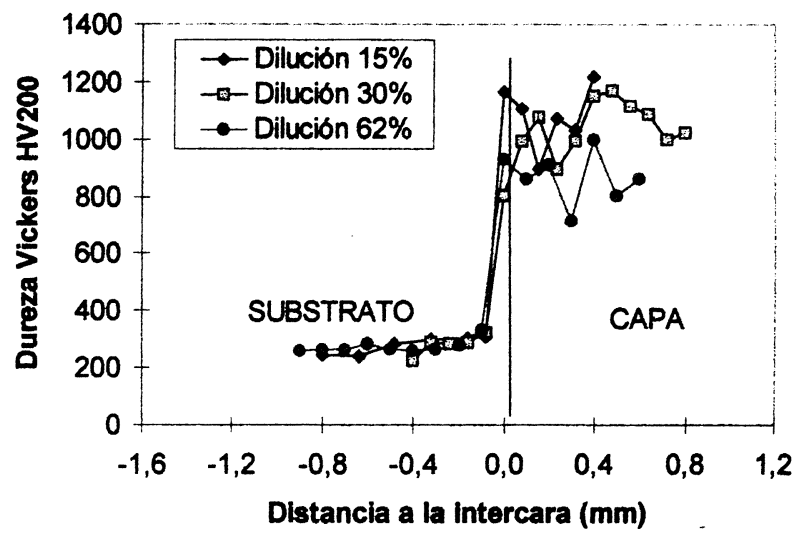

FIG. 7.- Microdurezas de tres capas de $83 \%$ WC-Co recargados por láser, con $15 \%, 30 \%$ y $62 \%$ de dilución.

FIG. 7.- Microhardness in $83 \%$ WC-Co laser cladded coatings, with different dilutions: 15, 30 and $62 \%$.

A)

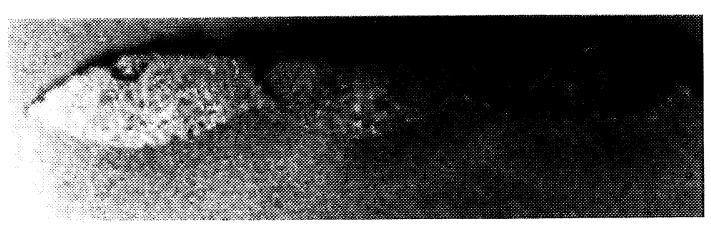

B)

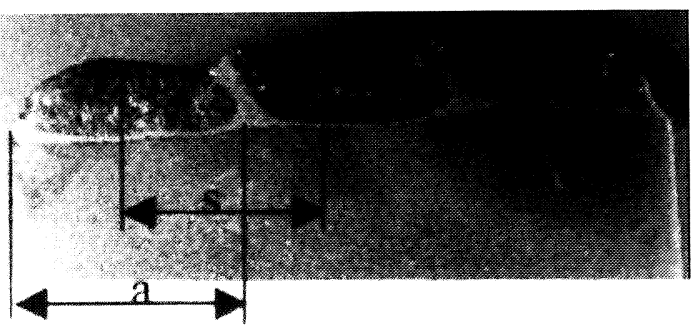

Fig. 8.- Capas de crC-NiCr realizadas con laser.

A) Con mucha dilución. B) Con poca dilución. (\% solapamiento $=(\mathrm{s} / \mathrm{a}) * 100)$.

FIG. 8.- CrC-NiCr laser cladded coating. A) With high dilution. B) With low dilution. (\% overlapping

$$
=(s / a) * 100) \text {. }
$$

zal focalizador del láser plantea una serie de problemas de operación y de resultados en las capas, que deben ser resueltos si se quiere alcanzar resultados satisfactorios.

La porosidad y el agrietamiento se atenúan con un ajuste adecuado de los parámetros de proceso, aunque su eliminación total no fue posible con los medios disponibles.

Los problemas surgidos con la inyección de polvo motivaron diversas modificaciones tanto en el inyector como en el sistema de protección de la óptica de focalización del láser (utilización de un segundo inyector de aire de protección), 
redundando finalmente en una mejora apreciable del rendimiento de la técnica.

En cuanto a la influencia de las variables en el proceso, se ha destacado el elevado número de variables que hay que considerar y la influencia que algunas de ellas, como el solapamiento de cordones, energía específica aportada, caudal de polvo y velocidad, tienen en la forma de los cordones y en la dilución. Finalmente, se demuestra que hay una energía por masa de polvo aportado umbral, que permite obtener diluciones próximas a cero y por debajo del cual el polvo no se adhiere al substrato.

\section{REFERENCIAS}

(1) Fouquet, F., Pelletier, J.M., Pilloz, M. y Vannes, A.B. Laser de puissance et Traitements des Materiaux. $1^{\mathrm{a}}$. Ed.
Presses Polytech. et Univ. Romandes. Lausanne (Suiza). 1991: 217

(2) Küpper, F., Gasser, A., Kreutz, E.W. y Wissenbach, K. Proc $3^{\text {rd }}$. European Conf. Laser Treat. Mater. Vol. 1. Erlangen (Alemania). 1990: 461-467

(3) Ramous, E., Giordano, L., Tiziani, A., Badan, B. y CAntello, M. Key Engineering Materials. Vols. 46 y 47. 1990: 425-434.

(4) Lugscheider, E. y Bolender, H. Proc $3^{\text {rd }}$. European Conf. Laser Treat Mater. Vol. 1. Erlangen (Alemania). 1990: 111-121.

(5) Cadenas Fdez, M. Tesis doctoral. Gijón (España) 1996.

(6) Marsden, C.F., Houdley, A.F.A. y Wagniere, J.D. Proc. $3^{\text {rd }}$. European Conf. Laser Treat. Mater. Vol. 1. Erlangen (Alemania). 1990: 543-553

(7) Nowotny, S., Techel, A., Luft, A. y Reitzenstein, W. Proc. ICALEO’93. 1993: 985-993. 